\title{
Street youth: Provision of medical care
}

$\mathrm{T}$ he article entitled "Chlamydia infection in street youth: Need for more aggressive screening programs" by Tam et al in this issue of the Journal (pages 49-52) once again highlights the problems associated with providing adequate medical care for street youth. In this article, the authors determined that $22 \%$ of the street youth in the Ottawa-Carleton region taking part in the study had serological evidence of previous Chlamydia trachomatis infection of which the youth involved had been unaware. Therefore, despite a nationwide emphasis directed towards screening and treatment of sexually transmitted (STD) and other diseases among street youth (1), a significant proportion of the youth in this study acquired at least one STD and received no therapy for it. This put them at risk for the long term consequences of this infection and allowed this infection to continue to spread in the general population unchecked. Chlamydia is one of a number of infections that street youth have an increased risk of acquiring; in regions where these infections are prevalent, street youth are also at increased risk of gonorrhea, human immunodeficiency virus (HIV), syphilis, human papilloma virus, tuberculosis, and hepatitis A, B and C. With the exception of hepatitis A, these infections all have the potential for long term medical complications. As well, this population can serve as a reservoir for these infections in the community, which significantly hampers public health efforts at disease control and elimination.

Adequate provision of medical care for street youth is complex. Street youth become street youth for a variety of reasons, and their willingness or ability to interact with the established medical system varies accordingly. Some may have fled their family because of violence or abuse, others may have been asked to leave the home because of uncontrollable behaviour, drugs or alcohol abuse, and others may find themselves homeless because of financial constraints within their family. The relationship each youth has with the medical establishment depends in part upon the reason the youth is on the street. Providing medical care to those fleeing from authority figures in the home, with underlying psychological problems or who regularly abuse drugs and alcohol is a major challenge. For this group, denial of symptoms or signs, unwillingness or inability to seek or follow medical advice, noncompliance with drug therapy and increased complication rates all significantly impair the delivery of health care. The issues of legal guardianship and ability to consent to care may delay provision of care for street youth who are under the age of 16 due either to reluctance of the child to seek care for fear of apprehension or to confusion concerning who is legally responsible for care and whether the child can legally consent to treatment. This problem often significantly interferes with HIV counselling and testing. Finally, financial constraints may result in poor compliance with medical advice for care because there may be no money to fill prescriptions, and those who are employed may feel that they are not able to take time off from work for follow-up care. Fortunately, with the present medical care system in Canada, the patient does not personally bear the cost of essential medical services, unlike in many other countries.

Is there a solution to this complex problem? The answer is probably both yes and no. This problem will likely continue until the social environment that leads to a significant number of youth leaving their homes to live on the streets changes. Children and adolescents have been running away from their homes for centuries, and the homeless have been with us since biblical times; an effective change of this nature thus seems unlikely. That does not mean that we cannot solve many of the problems of providing health care to these individuals, but there will not be one easy solution. There are several areas in which health care delivery can be improved for street youth.

Improvements in education concerning health care issues and sexual education in the school system at an age before most street youth leave school has been suggested by many and would likely have some benefit. However, as several studies of high risk youth attitudes and behaviour have shown, and as most clinicians who deal with this population are painfully aware, although most have adequate knowledge concerning the risks of various behaviours, street youth take the risks anyway. Rather than just an emphasis on education alone, we need to develop adequate counselling techniques that result in actual change of behaviour based on the knowledge provided. Successful research in this field would have benefits in many other medical health areas as well.

Access to health care is an area where improvements could more easily be made. Locating health care units in areas where street youth and the homeless reside is a logical approach. This allows individuals to walk to the facility rather than being dependent on transportation to and from appointments. We are all aware of the saying, "Out of sight, out of mind". The visual presence of the facility itself may also encourage individuals to seek help or it may act as a reminder to the individual to keep follow-up appointments. Clinics and hospitals in core areas need to be open for prolonged hours and need to be adequately staffed. Care givers should be those who want to work with this population, who are knowledgeable about the problems of these patients and who have exceptional interpersonal skills. Not everyone has the patience or the skill to interact appropriately with these patients. Because we as a society need and want these individuals to seek and receive appropriate health care, we need to eliminate as many of the barriers as possible.

Development of techniques for sample collection and for testing for multiple STD and other infections with minimal invasive techniques is also important. Speculum examina- 
tions take time and are embarrassing to many adolescent females, while most males are reluctant to have a first, much less a second, urethral swab specimen collected. Providing urine specimens for testing is more appealing to the patient. Use of polymerase chain reaction and related techniques will prove useful for diagnosis of multiple STDs with a single sample and will be helpful in more rapid diagnosis of other infections such as tuberculosis.

Single dose oral therapy for the more common STDs, gonorrhea and chlamydia, is now a possibility. This will eliminate noncompliance with treatment for these infections. There still remains the problem of providing long term treatment of tuberculosis, which may be improved by supervised therapy in a more 'user-friendly' clinical environment.

Unfortunately, cost constraints in the health care system are resulting in severe cutbacks and, in some areas, a trend towards centralization. This could adversely affect our ability to provide adequate medical care to this population. A system that provides emergency or crisis care only to this particular population is not in the best public interest. To quote a well known equestrian saying, "For want of a nail, the shoe was lost; for want of the shoe, the horse was lost." Therefore, throughout the upcoming health care reforms, we need to continue to emphasize the importance and cost effectiveness of preventive care and early diagnosis of infections and other illnesses.

\section{REFERENCE}

1. Gully PR, Bowie WR, MacDonald NE. Canadian guidelines for the prevention, diagnosis, management, and treatment of sexually transmitted diseases in neonates, children, adolescents, and adults. Can Commun Dis Rep 1992;18S1:1-210.

Joanne Embree MD FRCPC Pediatric Infectious Diseases/Medical Microbiology Health Sciences Centre Winnipeg, Manitoba 


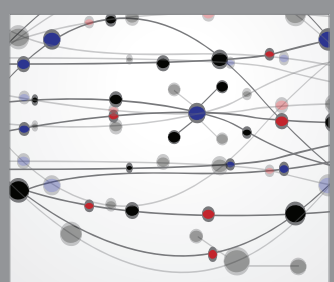

The Scientific World Journal
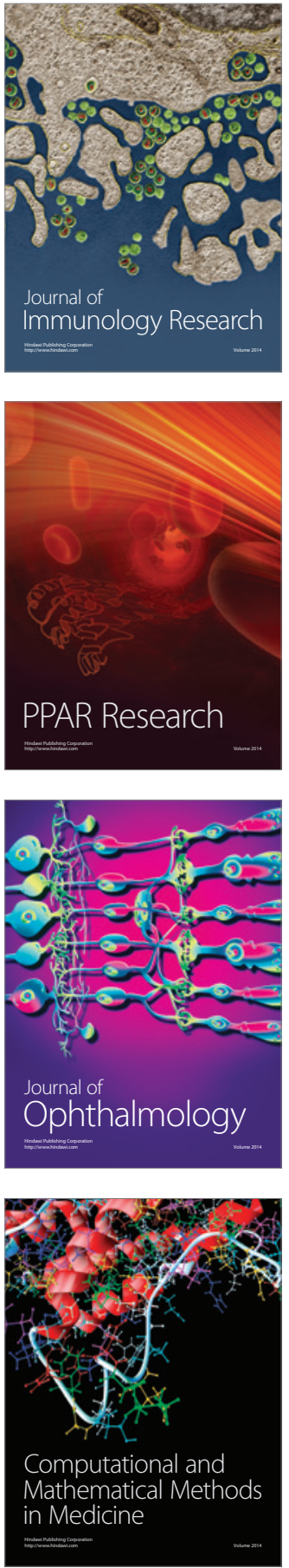

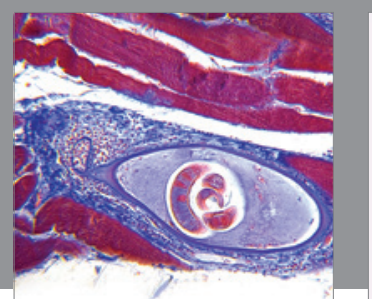

Gastroenterology Research and Practice

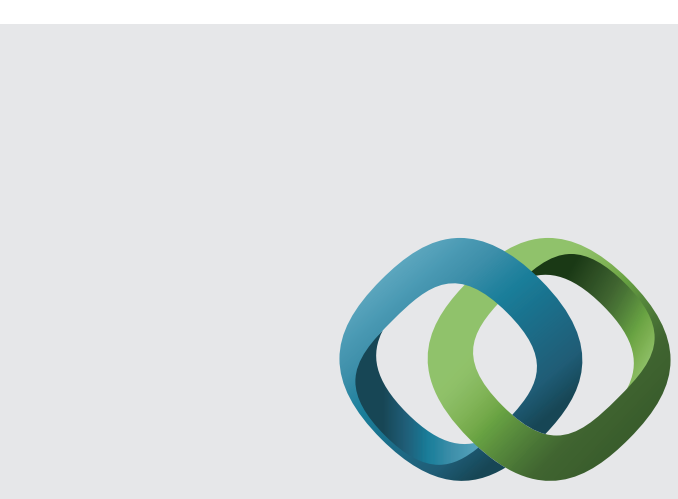

\section{Hindawi}

Submit your manuscripts at

http://www.hindawi.com
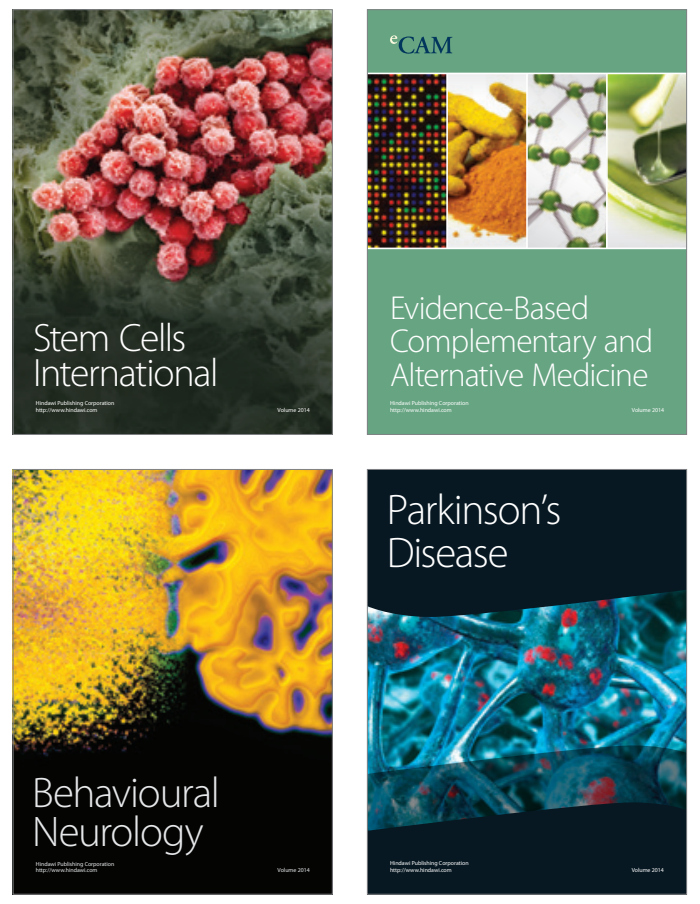
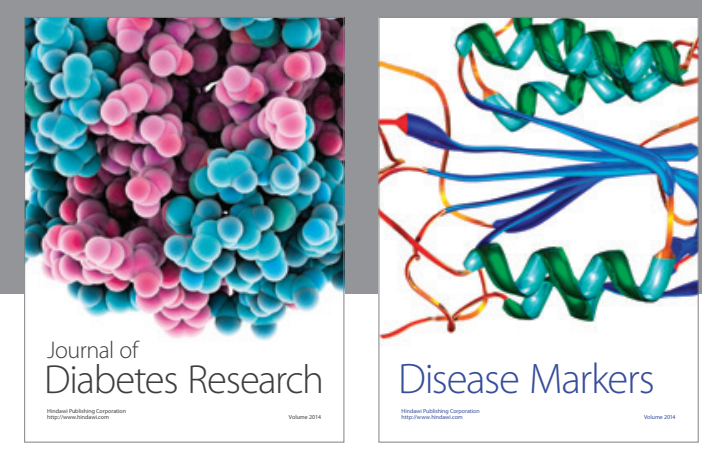

Disease Markers
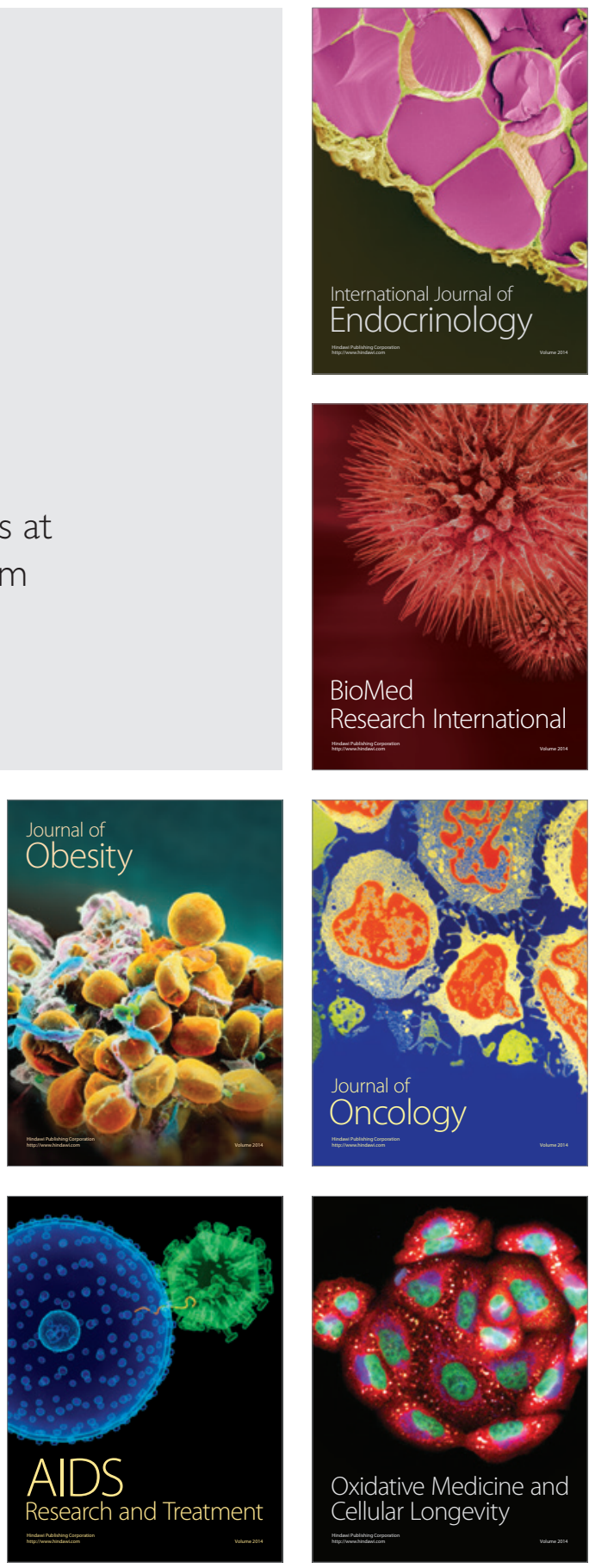